\title{
MgS in detached shells around carbon stars ${ }^{\star}$
}

\section{Mining the mass-loss history}

\author{
S. Hony ${ }^{1}$ and J. Bouwman ${ }^{2}$ \\ 1 RSSD-ESA/ESTEC, PO Box 299, 2200 AG Noordwijk, The Netherlands \\ 2 CEA, DSM, DAPNIA, Service d'Astrophysique, CEA Saclay, 91191 Gif-sur-Yvette Cedex, France
}

Received 30 June 2003 / Accepted 25 September 2003

\begin{abstract}
We investigate the dust composition of detached shells around carbon stars, with a focus to understand the origin of the cool magnesium-sulfide (MgS) material around warm carbon stars, which has been detected around several of these objects (Hony et al. 2002). We build a radiative transfer model of a carbon star surrounded by an expanding detached shell of dust. The shell contains amorphous carbon grains and $\mathrm{MgS}$ grains. We find that a small fraction of MgS dust ( $2 \%$ of the dust mass) can give a significant contribution to the IRAS $25 \mu \mathrm{m}$ flux. However, the presence of $\mathrm{MgS}$ in the detached shell cannot be inferred from the IRAS broadband photometry alone but requires infrared spectroscopy.

We apply the model to the detached-shell sources R Scl and U Cam, both exhibiting a cool MgS feature in their ISO/SWS spectra. We use the shell parameters derived for the molecular shell, using the CO submillimetre maps (Lindqvist et al. 1999; Schöier \& Olofsson 2001). The models, with MgS grains located in the detached shell, explain the MgS grain temperature, as derived from their ISO spectra, very well. This demonstrates that the MgS grains are located at the distance of the detached shell, which is a direct indication that these shells originate from a time when the stellar photosphere was already carbon-rich. In the case of R Scl, the IRAS photometry is simultaneously explained by the single shell model. In the case of U Cam, the IRAS photometry is under predicted, pointing to a contribution from cooler dust located even farther away from the star than the molecular shell.

We present a simple diagnostic to constrain the distance of the shell using the profile of the $\mathrm{MgS}$ emission feature. The emission feature shifts to longer wavelength with decreasing grain temperature. One can therefore infer a temperature and a corresponding distance to the star from the observed profile. Such a diagnostic might prove useful for future studies of such systems with SIRTF or SOFIA.
\end{abstract}

Key words. stars: AGB and post-AGB - stars: carbon - stars: mass-loss - stars: individual: R Scl \& U Cam circumstellar matter - infrared: stars

\section{Introduction}

The so-called "30" $\mu \mathrm{m}$ emission feature is commonly detected in the infrared (IR) spectra of carbon-rich (C-rich) evolved stars, from low to intermediate mass-loss asymptotic giant branch stars (AGB) through infrared carbon stars and post-AGB stars to planetary nebulae (e.g. Forrest et al. 1981; Omont 1993; Yamamura et al. 1998; Hony et al. 2002). The " $30 " ~ \mu \mathrm{m}$ feature, which exhibits itself as a prominent broad emission excess in the IR spectrum, extending from $\sim 24$ to $\sim 45 \mu \mathrm{m}$, is commonly attributed to magnesium-sulfide $(\mathrm{MgS})$ dust grains (e.g. Goebel \& Moseley 1985; Begemann et al. 1994). MgS grains are expected to form in C-rich

Send offprint requests to: S. Hony, e-mail: shony@rssd.esa.int

* based on observations obtained with ISO, an ESA project with instruments funded by ESA member states (especially the PI countries: France, Germany, the Netherlands and UK) with the participation of ISAS and NASA. environments (Lattimer et al. 1978; Lodders \& Fegley 1999), but are chemically unstable in oxidising, i.e. oxygen-rich, surroundings (Nuth et al. 1985). The feature profile varies considerably from source to source (e.g. Goebel \& Moseley 1985; Waters et al. 2000; Hrivnak et al. 2000). Hony et al. (2002) have presented a detailed spectral comparison between the emission expected from $\mathrm{MgS}$ grains and the profiles of the " 30 " $\mu \mathrm{m}$ emission feature found in the IR spectra of C-rich evolved stars obtained with the Short Wavelength Spectrograph (SWS, de Graauw et al. 1996) on board the Infrared Space Observatory (ISO, Kessler et al. 1996). Although there are also some profile variations that can be attributed to grain-shape variations, it was demonstrated that the main variations in the appearance of the " 30 " $\mu \mathrm{m}$ feature between distinct sources can be understood as a result of differences in the (average) $\mathrm{MgS}$ grain temperature. Conversely, one can derive the average grain temperature from the observed profile. One of the interesting findings of that study was the discovery of several "hot", 


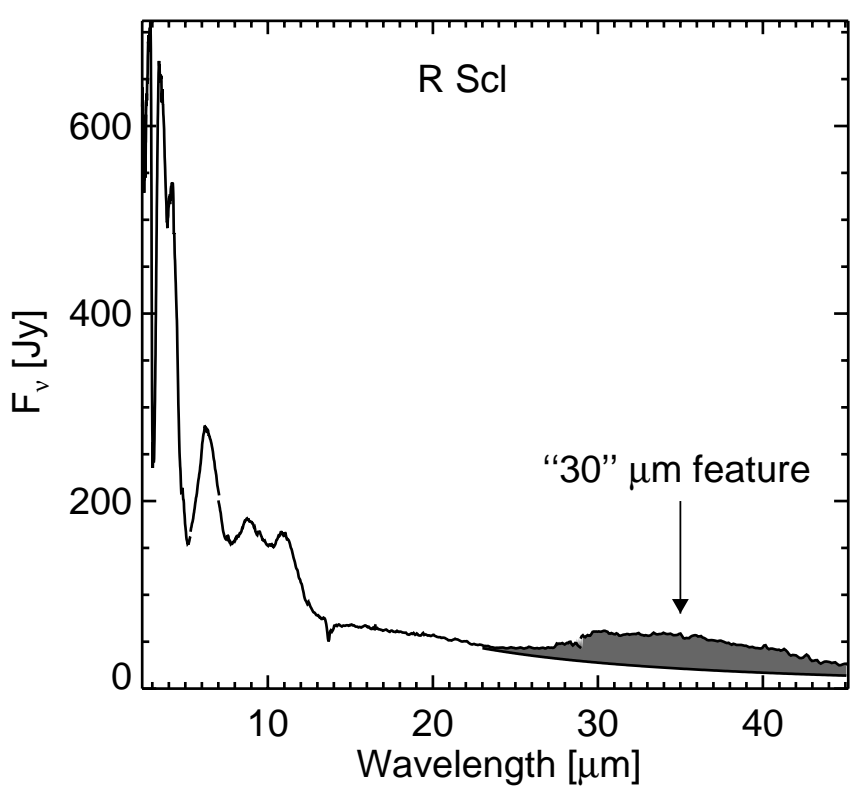

Fig. 1. The ISO/SWS spectrum of R Scl. An example of an optically bright carbon star that exhibits the " 30 " $\mu$ m feature attributed to cool MgS grains. The " 30 " $\mu \mathrm{m}$ feature is shaded in grey. At shorter wavelengths there are several prominent photospheric, molecular absorption bands. The emission band at $11.3 \mu \mathrm{m}$ is attributed to siliconcarbide grains.

i.e. optically bright, carbon stars that exhibit a cool $\mathrm{MgS}$ profile. In Fig. 1 we show the spectrum of R Scl. R Scl is an example of such an optically bright carbon star with a very prominent " 30 " $\mu \mathrm{m}$ feature. One of the most likely explanations for this phenomenon is the presence of $\mathrm{MgS}$ grains far from the star, whereas there is a lack of $\mathrm{MgS}$ grains closer by.

Some optically bright carbon stars are known to possess a detached shell of gas and dust around them (e.g. Waters et al. 1994; Schöier \& Olofsson 2001). In this paper, we explore the effect that $\mathrm{MgS}$ grains located in such a detached shell will have on the IR spectra and photometry of these stars. We build a grid of radiative transfer models of a carbon star surrounded by a detached dust shell which contains amorphous carbon (a-C) and $\mathrm{MgS}$ grains. We compare the synthetic spectra from these models with the IRAS photometry of known carbon stars. We further apply the model to two specific carbon stars: R Scl and U Cam. The detached shells around these sources are clearly resolved in molecular line emission (Schöier \& Olofsson 2001) and good quality ISO/SWS spectra are available to compare with the synthetic spectra. This allows us to address the question on whether the $\mathrm{MgS}$ grains are located at the same distance as these molecular shells.

\subsection{The origin of the dust far from the star}

Carbon stars are known to loose large amounts of envelope material through a dust driven wind. The observational indications that this mass loss may occur in a non-steady fashion are important for the present study. Perhaps the most complete picture arises from the IRAS colour-colour diagram (van der Veen \& Habing 1988) of C-rich evolved stars (Fig. 2). This diagram

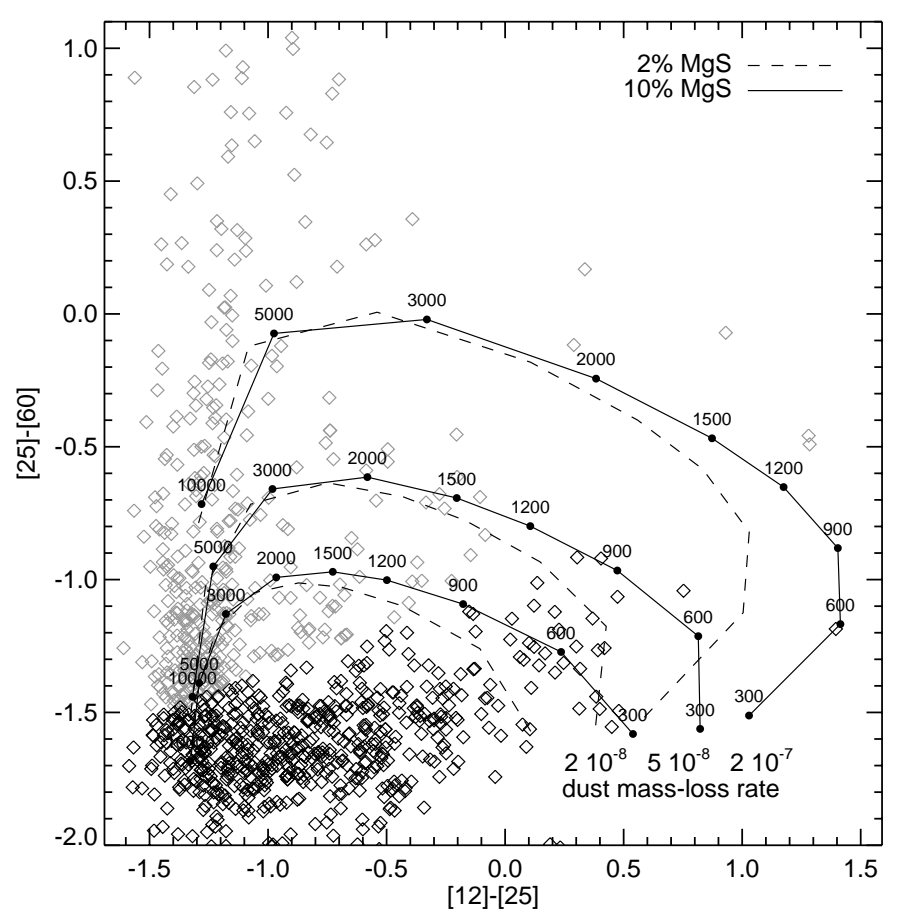

Fig. 2. IRAS colour-colour diagram of galactic carbon stars (diamonds). Stars that exhibit a single temperature SED are shown in black. Stars with a far-IR excess are grey. We also show the tracks that the models follow in the diagram. The tracks are for a mass-loss burst lasting for 200 years. The dust mass-loss rate increases from the bottom to the top curves $\left(2 \times 10^{-8}-2 \times 10^{-7} M_{\odot} / \mathrm{yr}\right)$. The time elapsed since the burst is indicated in the figure. The difference between the dashed and the full lines is the relative amount of MgS. At the onset of the burst, when the dust is warmest, the contribution of $\mathrm{MgS}$ makes the tracks differ substantially.

shows the $60 \mu \mathrm{m}$ over $25 \mu \mathrm{m}$ flux-ratio (expressed as a magnitudes difference) versus the $25 \mu$ m over $12 \mu$ m flux-ratio based on the IRAS photometry. A star's position in this diagram is thus a measure of the shape of its IR spectral energy distribution (SED). The diagram shows that most of the carbon-star SEDs are dominated by a single temperature. However, about one third of them show evidence of a second, cooler dust component. This cooler dust component manifests itself through a far-IR excess.

The common interpretation is that this excess is due to a detached dust shell located away from the star (e.g. Willems \& de Jong 1988; Zuckerman 1993). This shell originates from a previous epoch of mass loss, when the mass-loss rate exceeded the present day mass-loss rate by several factors. Although there has been some discussion in the literature on whether this far-IR excess is actually related to the carbon stars (Ivezic \& Elitzur 1995; Egan et al. 1996), several of these detached shells have now been directly detected. These shells have been resolved either in the IR (Young et al. 1993; Waters et al. 1994; Izumiura et al. 1995, 1996, 1997; Izumiura \& Hashimoto 2000) or in molecular lines in the submillimetre domain (Yamamura et al. 1993; Olofsson et al. 1996; Lindqvist et al. 1999; Schöier \& Olofsson 2001). In a few cases, the optical light scattered by the material in these shells has been directly detected (Izumiura et al. 2000; González Delgado et al. 2001). 
The origin of these shells, i.e. the cause of the change in mass-loss rate, is matter for debate. The earliest suggestion was that occurrence of these shell was closely related to the photospheric composition of the AGB star. Specifically, it was suggested that these shells arise as a result of the thermal pulse, that increased the number ratio of $\mathrm{C}$-atoms to $\mathrm{O}$-atoms in the photosphere of the AGB star from less than unity (O-rich) to larger than unity (C-rich) (Willems \& de Jong 1988; Chan \& Kwok 1988). Later studies showed that these shells may arise due to thermal pulses in general, as long as the star is close to the tip of the AGB, i.e. with a luminosity close to the critical luminosity (e.g. Schröder et al. 1998; Steffen \& Schönberner 2000). Recent numerical simulations of the momentum transfer in the dust driven winds of AGB stars show that modulations in the frictional coupling between the gas and the dust can also cause non-steady mass loss and shell formation (Simis et al. 2001). These results have been obtained for a C-rich gas and dust composition and it is at present not clear whether the same mechanism applies to O-rich environments.

\subsection{The composition of the material in the shell}

Closely related to the question of the origin of these detached shells is the question of their composition. The molecular and dust composition in the shells is a tracer of the chemical and physical conditions, in particular the $\mathrm{C} / \mathrm{O}$ number ratio, in the atmosphere of the star at the time the shell was formed. There are various indirect evidences that these shells are C-rich. Bujarrabal \& Cernicharo (1994) find that the molecular line ratios for the various molecules are indicative of a C-rich chemistry. From modelling of the SED, one also tends to find that the opacities of C-rich dust agree better with the observed far-IR excesses than O-rich dust (e.g. Bagnulo et al. 1997). However these models are not conclusive and models with O-rich dust can also be fitted to the SEDs. The possibility of detecting $\mathrm{MgS}$ grains in these shells is a tantalising one, since this gives us a direct handle on the prevailing $\mathrm{C} / \mathrm{O}$ ratio at the time the matter was ejected. This is due to the fact that the " 30 " $\mu \mathrm{m}$ feature, attributed to $\mathrm{MgS}$ grains, is only found in C-rich environments and has, until now, not been detected in O-rich environments.

The paper is organised as follows. In Sect. 2 we present the description of the model. The results of the model are presented and compared to IRAS observations in Sect. 3. We apply the model to R Sculptoris and U Camelopardalis in Sect. 4. Both these sources have a " 30 " $\mu \mathrm{m}$ feature in their ISO/SWS spectra and the detached gas shells have been spatially resolved with molecular line observations. This allows us to test whether the molecules and dust are co-spatial. Finally, in Sect. 5, we summarise our findings and discuss future prospects.

\section{Model description}

Our model consist of a single spherical dust shell surrounding a carbon star with a low current day mass-loss rate. The radiation of the star is partially absorbed by the dust grains in the shell. The absorbed light heats the dust grains, which subsequently emit light at IR wavelengths.
The optically visible C-star is represented by a Planck function of $2500 \mathrm{~K}$. This temperature is representative for the ensemble of optically bright carbon stars with cold $\mathrm{MgS}$ grains in the ISO/SWS sample from Hony et al. (2002), where we use the effective temperatures as given by Bergeat et al. (2001) The luminosity of the star is set to $10000 L_{\odot}$, which corresponds to a stellar radius of $535 R_{\odot}$. Of course, the whole system can simply be scaled to a different luminosity for the central star by scaling all dimensions with the appropriate value.

\subsection{Shell parameters}

We assume a constant expansion velocity, with a typical value of $15 \mathrm{~km} \mathrm{~s}^{-1}$. The expansion velocity is constant. During the burst that produced the shell, the mass-loss rate was constant. Before and after the burst the mass loss was negligible. Due to the expansion, the dimensions of the shell are related to the time since termination of the burst as:

$R_{\text {in }}=R_{\star}+v_{\text {exp }} * t$ and $R_{\text {out }}=R_{\text {in }}+v_{\text {exp }} * \Delta t$,

where $R_{\star}$ is the radius of the star, $R_{\text {in }}$ and $R_{\text {out }}$ are the inner and outer edges of the dust shell after time $t$. $\Delta t$ is the duration of the high mass loss. We calculate synthetic spectra for times after ejection $(t)$ from 300 to 10000 year. For $t<300$ year the optical depth through the dust shell is very large and these systems will look essentially like high mass-loss carbon stars, instead of detached-shell sources. For $t>10000$ year the $\mathrm{MgS}$ grains are too cold to have any detectable signature in the resulting SED. We vary $\Delta t$ between 200 to 1500 year. The corresponding values for the shell thickness $\left(1 \times 10^{16}-7 \times 10^{16} \mathrm{~cm}\right)$ bracket the thickness of the detached shells derived in Schöier \& Olofsson (2001)

The gas density in the shell follows from $\rho_{\text {gas }}(R)=$ $\dot{M} /\left(4 \pi R^{2} v_{\text {exp }}\right)$, where $\rho_{\text {gas }}$ is the gas density as a function of radius $(R)$ and $\dot{M}$ is the mass-loss rate. The dust density ( $\left.\rho_{\text {dust }}\right)$ is important for the radiative transfer calculations. This value is the ratio of the gas density to the gas-to-dust mass ratio $(\Psi)$, which implies that the models are determined by the dust mass-loss rate $\left(\dot{M}_{\text {dust }}=\dot{M} / \Psi\right)$. We calculate model spectra for the dust mass-loss rate in the range $2 \times 10^{-9}-2 \times$ $10^{-7} M_{\odot} / \mathrm{yr}$. This translates to a total mass loss-rate in the range $5 \times 10^{-7}-10^{-4} M_{\odot} / y r$, using a typical value of $\Psi=250$. However, it is not clear, whether such a standard value is always applicable (see also Sect. 4).

\subsection{Dust parameters}

We use a-C and $\mathrm{MgS}$ as the composition of the dust. The optical properties of a-C are taken from Preibisch et al. (1993). We use an a-C grain-size distribution according to: $n(a) \propto$ $a^{-3.5}$ with $0.01 \mu \mathrm{m} \leq a \leq 1.0 \mu \mathrm{m}$, where $n(a)$ is the number density of grains with radius $a$. For the $\mathrm{MgS}$ grains we use the same optical properties used to fit the observations of the C-rich post-AGB star HD 56126 (Hony et al. 2003). These properties are based on the calculated absorption cross-sections in the IR, using the optical constants as published by Begemann et al. (1994) We calculate the IR absorption cross-sections for 

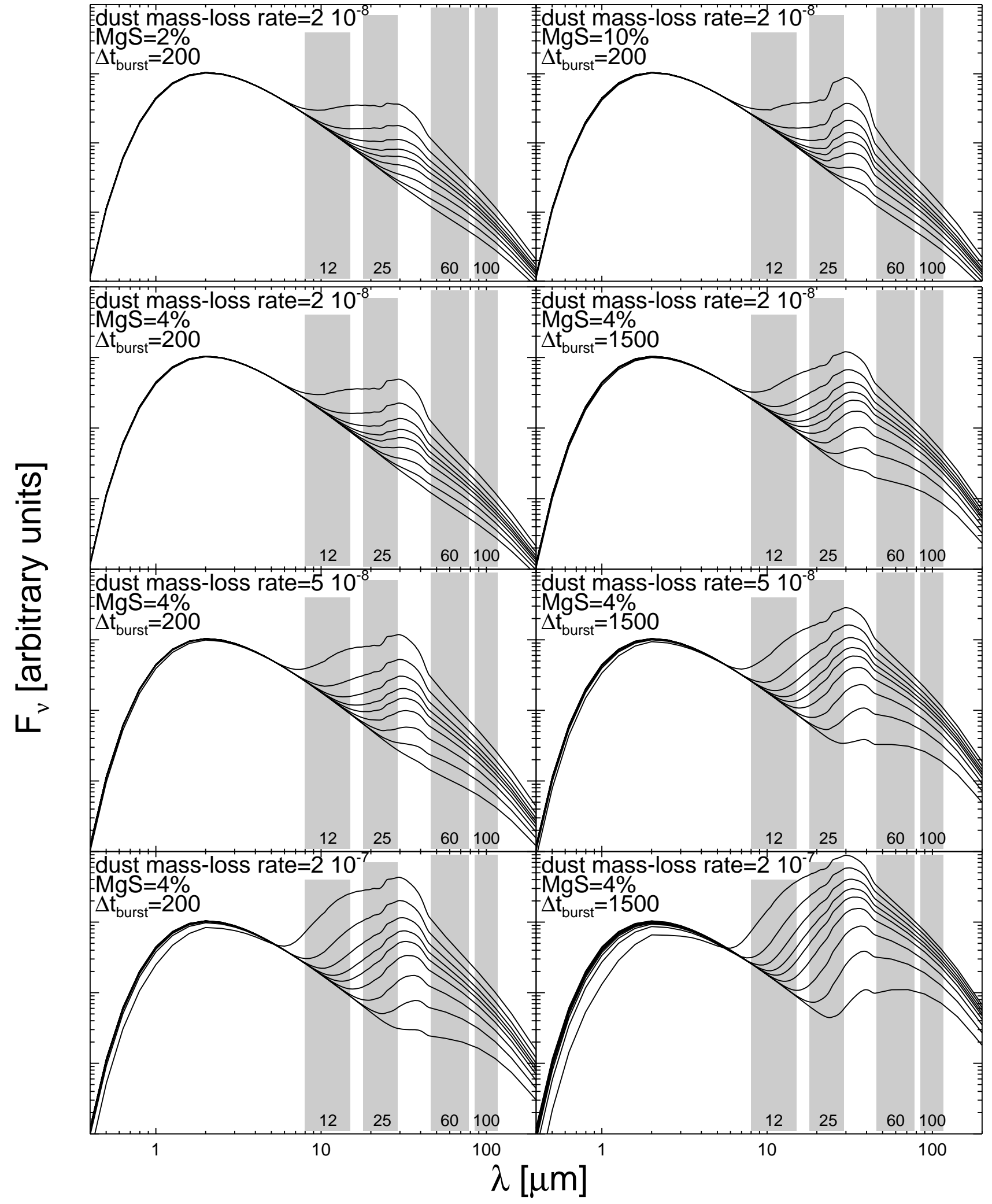

Fig. 3. Synthetic SEDs from the expanding dust shell containing amorphous carbon and MgS grains. In each panel, the curves from top to bottom represent the system 300, 600, 900, 1200, 1500, 2000, 3000, 5000 and 10000 year after ejection of the shell. The top panels show the effect of varying the percentage of $\mathrm{MgS}$ grains. In the bottom six panels, the mass-loss rate increases from top to bottom and the duration of the burst increases from left to right. Also indicated are the wavelength regions that contribute to the different IRAS broadband filters. The $\mathrm{MgS}$ grains are the cause of the " 30 " $\mu \mathrm{m}$ feature, the broad extra emission band between $\sim 23$ and $\sim 45 \mu \mathrm{m}$. 
a continuous distribution of ellipsoids shape-distribution. This distribution results in a resonance which agrees best with the observed feature (e.g. Begemann et al. 1994; Szczerba et al. 1999; Hony et al. 2002). Unfortunately, the optical properties of $\mathrm{MgS}$ have not been measured below $10 \mu \mathrm{m}$. We assume a constant absorption cross-section below $1 \mu \mathrm{m}$ of $\pi \times 10^{-4} \mu \mathrm{m}^{2}$ for a grain radius of $0.01 \mu \mathrm{m}$. We further assume a linear decrease in the absorption cross-section to 0 from 1 to $2 \mu \mathrm{m}$ and 0 between 2 and $10 \mu \mathrm{m}$. The cross-section assumed here is identical to the one used to model HD 56126. The location of the dust around that star is well determined from extensive IR imaging studies (e.g. Dayal et al. 1998; Jura et al. 2000; Kwok et al. 2002) as the dust shell is clearly resolved. The assumed absorption cross-section below $1 \mu \mathrm{m}$ yields a $\mathrm{MgS}$ grain temperature, at the location of the dust shell of HD 56126, which agrees well with its SWS spectrum (Hony et al. 2003).

We vary the relative proportion of $\mathrm{MgS}$ to a-C from 2 to 10 percent by mass. A smaller fraction of $\mathrm{MgS}$ will not produce a significant " 30 " $\mu \mathrm{m}$ feature. A larger fraction of $\mathrm{MgS}$ is unlikely to be present because the amount of $\mathrm{MgS}$ grains is limited by the abundance of $\mathrm{Mg}$ and $\mathrm{S}$ atoms. For a solar composition gas, the maximum mass contained in $\mathrm{Mg}+\mathrm{S}$ pairs relative to the mass of the $\mathrm{C}$-atoms is 20 percent. However, not all available $\mathrm{Mg}, \mathrm{S}$ and $\mathrm{C}$ atoms are necessarily be condensed into dust grains.

\subsection{Dust radiative transfer code}

We use the proprietary dust radiative transfer code MODUST. This code solves the monochromatic radiative transfer equation, from UV/optical to millimetre wavelength, in spherical geometry subject to the constraint of radiative equilibrium, using a Feautrier type solution method (Feautrier 1964; Mihalas 1978). This yields the temperature of the dust grains. The code allows to have several different dust components of various grain sizes and shapes. We refer to Bouwman et al. (2000) and Bouwman (2001) for a description of techniques used in MODUST. MODUST yields both the model SED and intensity maps. From the obtained model spectra and intensity maps, it is straightforward to simulate observations. For example, we simulate the synthetic SWS spectra by overlaying the apertures as a function of SWS subband, as listed in de Graauw et al. (1996), on the intensity maps. IRAS broadband fluxes are simulated by convolving the spectra with the IRAS bandpasses (Beichman et al. 1988). We solve the transfer equations at 36 wavelengths, on a logarithmically spaced wavelength grid, from 0.2 to $1000 \mu \mathrm{m}$. This is sufficient for correctly integrating over the wavelength, when performing the radiative equilibrium calculation. These wavelengths also cover the entire SED. In addition, we calculate the synthetic mid-IR spectrum from 2 to $45 \mu \mathrm{m}$, with linear wavelength steps of $0.5 \mu \mathrm{m}$, to compare with the observed SWS spectra.

\section{Results}

In Fig. 3, we show a subset of the SEDs that result from our model. We show the main effects of varying the mass-loss rate, the fraction of $\mathrm{MgS}$ grains and the duration of the burst. We also indicate which part of the SED contributes to the various IRAS photometric filters. There are a few general points that emerge from the analysis.

1. The " 30 " $\mu \mathrm{m}$ feature is detectable even when present at a concentration of only 2 percent. This is especially true shortly after the ejection, when the $\mathrm{MgS}$ grains are relatively warm and the spectra clearly exhibit the characteristic onset at $\sim 26 \mu \mathrm{m}$.

2. MgS contributes considerably to the IRAS $25 \mu \mathrm{m}$ flux during the first 2000 year when it is warm enough to emit within the IRAS $25 \mu \mathrm{m}$ bandpass.

3. The shell becomes quickly optically thin for the radiation of the central star and therefore the emission of $\mathrm{MgS}$ scales with the amount of $\mathrm{MgS}$ in the shell.

4. The $12 \mu \mathrm{m}$ excess disappears within $\sim 750$ year.

5. As expected, the IR excess increases with larger shell mass and is more pronounced for a short fierce burst than for a prolonged ejection at a lower mass-loss rate, even for the same amount of total material shed.

\subsection{The IRAS colour-colour diagram}

In Fig. 2 we compare the tracks as predicted from our model with the IRAS colours of known galactic carbon stars. The diagram is constructed in the following way. We cross-correlate the positions of the sources in the General Catalog of Galactic Carbon Stars (Alksnis et al. 2001) with the sources within 30" in the IRAS point source catalogue (Joint IRAS Science Working Group 1988). This yields 3417 associations. From these sources we only use those with a relative flux uncertainty less than 50 percent in the IRAS 12, 25 and $60 \mu \mathrm{m}$ filter. In Fig. 2, the maximum uncertainties are 0.55 and 0.6 and the average uncertainties are 0.11 and 0.15 in [12]-[25] and [25]-[60], respectively.

The tracks explain the general features of the observed colour-colour diagram well. The colours of the systems immediately after ejection of the shell are similar to the colours of the infrared carbon stars, because of the large optical depth in the shell. The expansion of the shell causes the optical depth to rapidly decrease and the $12 \mu \mathrm{m}$ excess to diminish as the dust becomes cooler. Therefore, the source moves rapidly to the left in the diagram. The stars spend by far the longest time on the vertical track, at [12]-[25] $\approx-1.2$, while the $60 \mu \mathrm{m}$ excess due to the cool dust slowly diminishes. This explains the clustering of the stars in this region of the diagram. The effect of the " 30 " $\mu \mathrm{m}$ feature due to $\mathrm{MgS}$ grains can only be noticed within 2000 years after ejection. However, the influence of $\mathrm{MgS}$ on the colours is not unique, because the effect of the $\mathrm{MgS}$ grains in the colour-colour diagram can be mimicked by a higher mass-loss rate or a longer burst duration. This implies that, due to the presence of MgS grains in these shells, and thus of the " 30 " $\mu \mathrm{m}$ feature, the mass in the shell may be overestimated, when determined from the IRAS photometry alone.

We note that there are several carbon stars in the IRAS database with $60 \mu \mathrm{m}$ excesses larger than the ones shown in Fig. 2. We have not attempted to reproduce these 


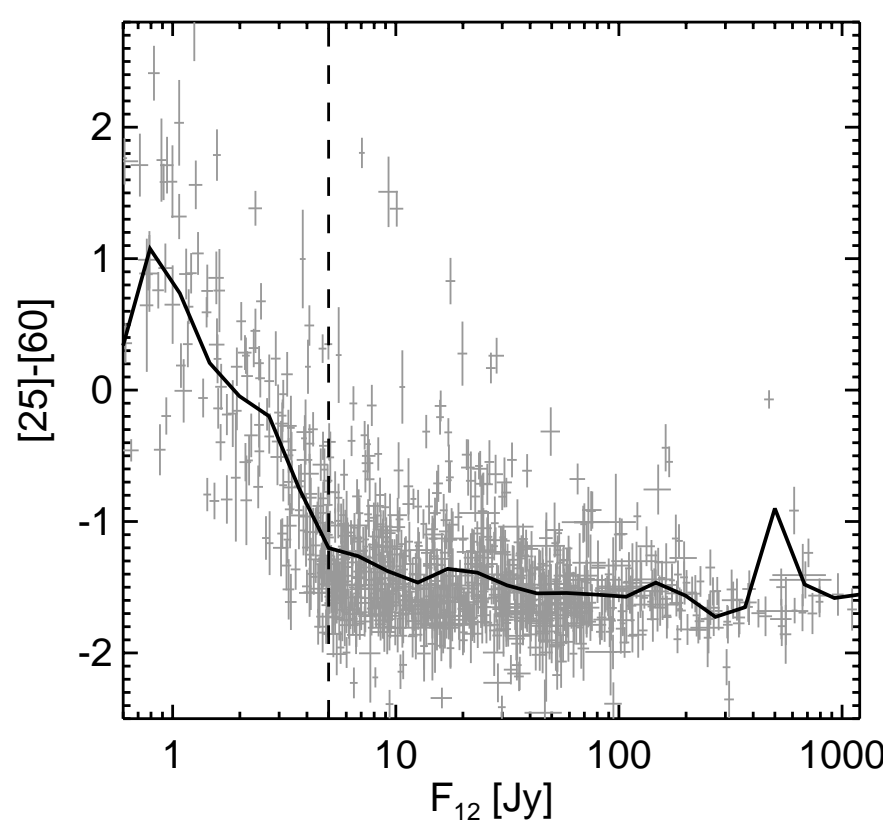

Fig. 4. IRAS colour-magnitude diagram of galactic carbon stars. The thick black line shows the mean of the [25]-[60] colour as a function of $12 \mu \mathrm{m}$ flux for the carbon stars (shown in grey). The sources with the largest $60 \mu \mathrm{m}$ excess are all very faint at $12 \mu \mathrm{m}$ (to the left of the dashed line). The photometry of these sources is probably affected by cirrus contamination or confusion due to the poor angular resolution of IRAS.

IRAS colours with our model. It is likely that there is a sizeable contamination of the far-IR fluxes among the carbon stars, as has been argued by Ivezic \& Elitzur (1995). Specifically, we point out that the largest IRAS excesses are found for the sources with low $12 \mu$ m fluxes, i.e. sources far away with a much larger chance for confusion or cirrus contamination. The carbon stars with IRAS colour [25]-[60] $>0$ are almost exclusively found among sources with $F_{12}<5$ Jy (see Fig. 4). In this paper, we are only concerned with the sources that have $[25]-[60] \lesssim 0.5$ and clearly have a detached shell associated with them. In order to obtain $60 \mu \mathrm{m}$ excesses in the range $[25]-[60] \simeq 1$ or even higher (see Fig. 4), we have to increase the total dust mass to $10^{-4} M_{\odot}$, which in turn yields an estimated mass-loss rate an order of magnitude higher than those derived for the best studied cases. It is not clear whether the same detached-shell model can be invoked to explain such extreme excesses.

\section{2. $\mathrm{MgS}$ as a temperature/distance diagnostic}

Because the shell becomes rapidly optically thin, we can give an approximate temperature of the $\mathrm{MgS}$ grains as a function of shell radius and effective temperature of the star. The effects of the expansion of the shell are shown in Fig. 6. We show two curves. The diamonds show the dependence of temperature on distance. The squares show how this temperature translates into a shifting of the " $30 " \mu \mathrm{m}$ feature (see also Fig. 3). The shift is expressed in the centroid position of the $\mathrm{MgS}$ feature, i.e. the wavelength which divides the feature in equal energy halves, see also Fig. 5. This quantity is in general more easily

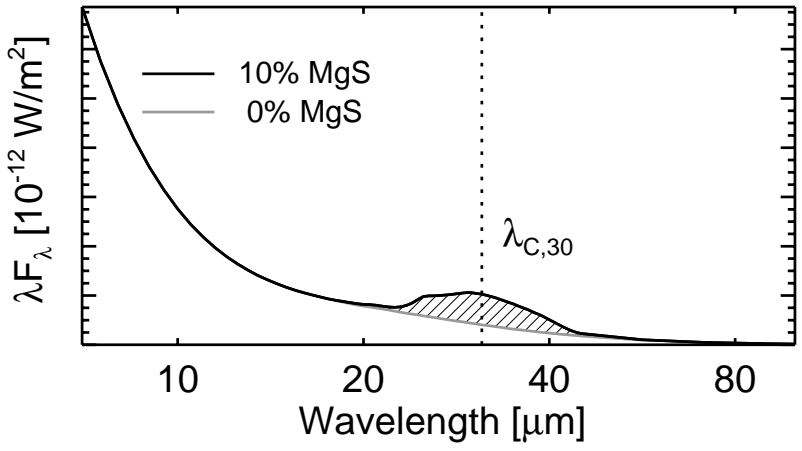

Fig. 5. Illustration of the definition of the centroid position of the "30" $\mu \mathrm{m}$ feature. We shows two model spectra of a detached shell containing 0 and 10 percent $\mathrm{MgS}$. The difference is the " 30 " $\mu \mathrm{m}$ feature due to the presence of the $\mathrm{MgS}$ grains. On this scale equal surface corresponds to equal energy. The centroid position is indicated by the dashed line, which divides the " 30 " $\mu \mathrm{m}$ feature into equal surface.

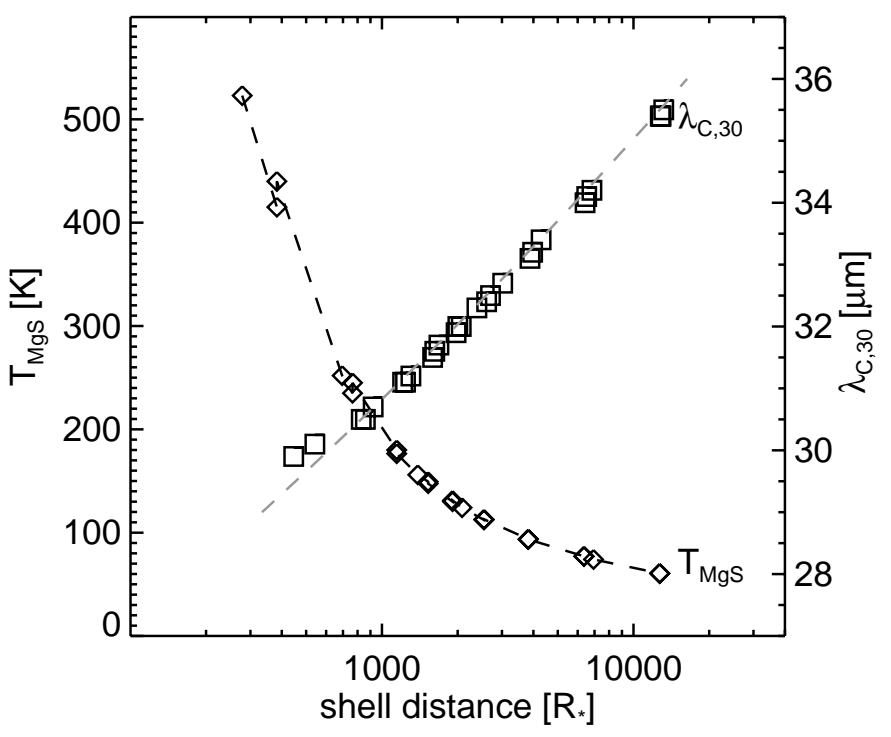

Fig. 6. $\mathrm{MgS}$ temperature and the centroid position of the " 30 " $\mu \mathrm{m}$ feature as a function of distance to the star. The diamonds show the $\mathrm{MgS}$ temperature at the inner edges of the detached dust shell. The squares represent the centroid position of the " 30 " $\mu \mathrm{m}$ emission feature versus the typical distance of the shell, which is defined as the inner radius plus one quarter of the shell thickness. The grey dashed line shows a simple analytic representation of the relation between the distance and the centroid position (see Eq. (2)).

derived from the spectra and is also a better diagnostic of the temperature than the peak position. For the distance of the shell we take a "typical" distance, defined as the inner radius plus one quarter of the shell thickness. This is to account for the fact that the dust on the inside of the shell is hotter and thus brighter than on the outside. The points shown in Fig. 6 are for shell thickness between 250 and $2000 R_{\star}$, which corresponds to a burst duration from 200 to 1500 years. An approximate powerlaw expression (valid for $30<\lambda_{\mathrm{C}, 30}<36 \mu \mathrm{m}$ ) is given by:

$\frac{R_{\text {shell }}}{R_{\star}}=\left(\frac{\lambda_{\mathrm{C}, 30}}{21 \mu \mathrm{m}}\right)^{18} \times\left(\frac{T_{\star}}{2500 \mathrm{~K}}\right)^{2}$, 
Table 1. Shell parameters of R Scl and U Cam.

\begin{tabular}{|c|c|c|c|c|c|c|}
\hline \multirow[b]{2}{*}{ Source } & \multirow[b]{2}{*}{$\begin{array}{l}\mathrm{d} \\
{[\mathrm{pc}]}\end{array}$} & \multicolumn{3}{|c|}{ Gas shell $^{a}$} & \multicolumn{2}{|c|}{ Dust shell } \\
\hline & & $\begin{array}{c}R_{\text {in }} R_{\text {out }} \\
\quad\left[{ }^{\prime \prime}\right]\end{array}$ & $\begin{array}{l}R_{\text {in }} R_{\text {out }} \\
{\left[10^{16} \mathrm{~cm}\right]}\end{array}$ & $\dot{M}$ & $\begin{array}{l}\dot{M}_{\text {dust }} \\
/ \mathrm{yr}]\end{array}$ & $\begin{array}{l}\mathrm{MgS} \\
\%\end{array}$ \\
\hline $\mathrm{R} \mathrm{Scl}$ & 360 & 1326 & $\begin{array}{ll}7 & 14\end{array}$ & $4 \times 10^{-6}$ & $8 \times 10^{-9}$ & 4 \\
\hline $\mathrm{U} \mathrm{Cam}^{b}$ & 500 & 79 & 5.56 .6 & $9 \times 10^{-6}$ & $1.6 \times 10^{-8}$ & 4 \\
\hline
\end{tabular}

${ }^{a}$ Taken from Schöier \& Olofsson (2001).

${ }^{b}$ The dust parameters for U Cam only concern the dust located inside the SWS aperture (see text for details).

where $R_{\text {shell }}$ is the typical distance of the shell. Note that, in physical terms, the shape of the emission feature is a weighted sum of Planck functions, depending on the $\mathrm{MgS}$ grain temperatures, convolved with the wavelength dependent $\mathrm{MgS}$ absorption cross-section. Therefore, the simple power-law expression and the constants in the first term of Eq. (2) lack a direct physical interpretation. With optical photometry of the central star, both $T_{\star}$ and $R_{\text {shell }}$ (in arcsec) can be determined independent of distance, as the angular size of the star $\left(R_{\star}\right.$ in $\left.\operatorname{arcsec}\right)$ is set by $T_{\star}$ and the photometry alone. How practical Eq. (2) will be in reality remains to be demonstrated, because it is known that there are also some differences in the shape of " 30 " $\mu \mathrm{m}$ feature between sources that are not caused by temperature but are more likely due to the grain-shape distribution (Hony et al. 2002). Such a difference can shift $\lambda_{\mathrm{C}, 30}$ by up to $2.5 \mu \mathrm{m}$ : a factor 4 in shell radius! Because the main deviation in the profile is a $26 \mu \mathrm{m}$ excess, the tendency will be to find a lower value of $\lambda_{\mathrm{C}, 30}$ and therefore derive a too small shell radius.

\section{Application to sources with ISO/SWS spectra}

In the previous section we have shown that the presence of $\mathrm{MgS}$ in the detached shells can have a significant effect on the IRAS $25 \mu \mathrm{m}$ photometry. However, the presence of $\mathrm{MgS}$ grains in the shell cannot be inferred from the IRAS photometry alone. In this section we focus on those warm carbon stars that show a "30" $\mu \mathrm{m}$ feature in their SWS spectrum.

\subsection{Application to R Sculptoris}

We first apply the model to R Scl. This star is among the best studied optically bright carbon stars with a far-IR excess. Its detached shell has been resolved in molecular lines emission (Olofsson et al. 1996) and scattered light from the detached shells is detected (González Delgado et al. 2001). Furthermore, $\mathrm{R}$ Scl has the strongest " 30 " $\mu \mathrm{m}$ feature of the optically bright carbon stars observed with SWS.

For the parameters of the dust shell (see Table 1), we take the inner and outer edge of the shell of the detached gas shell as listed by Schöier \& Olofsson (2001, their Table 6). These parameters are derived from mapping observations of the molecular CO emission, in which the shell is clearly resolved. We use the same distance to the star as these authors do, to facilitate a direct comparison of the parameters, which they derive and ours. With the inner and outer edge of the shell fixed, we have only two free parameters: the dust mass-loss rate and the mass fraction of $\mathrm{MgS}$. We vary these parameters and compare the results with the IRAS and ISO observations. The strength of the IR continuum excess is set by the amount of amorphous carbon in the shell, which scales linearly with the dust massloss rate. The fraction of $\mathrm{MgS}$ influences the strength of the resultant " 30 " $\mu \mathrm{m}$ feature. The best agreement with the observations is found with 4 percent of $\mathrm{MgS}$ and a dust mass-loss rate of only $8 \times 10^{-9} M_{\odot} /$ year. This best fit is shown in Fig. 7 . We derive a total dust mass in the shell, of $1.2 \times 10^{-5} M_{\odot}$. The gas mass as determined by Schöier \& Olofsson (2001) is $5.4 \times 10^{-3} M_{\odot}$, which yields for the gas-to-dust mass ratio: $\Psi=450$. The model parameters are given in Table 1 .

We show a sketch of the model, with the dimensions of the relevant SWS apertures and the IRAS beam-sizes overlaid, in Fig. 8. The IRAS measurements sample the complete detached shell, whereas the SWS spectrograph detects only a fraction of the shell. The different regions probed by the various instruments give rise to differences in the flux levels measured by these instruments. The IRAS measurements sample the complete shell and should therefore yield much higher flux level than SWS does. This is indeed as observed (see Fig. 7). A smaller difference in the flux level, due to the size of the SWS apertures, is also observed in the SWS data below $27.5 \mu \mathrm{m}$ and above $29 \mu \mathrm{m}$.

The model is able to explain the strength of the MgS resonance within the SWS aperture accurately. It simultaneously explains the much stronger excess in the IRAS beam. The presence of the $\mathrm{MgS}$ excess was already suspected by Bagnulo et al. (1998) who found that the IRAS $25 \mu \mathrm{m}$ photometry was higher than expected on the basis of the flux level of their Cooled Grating Spectrograph (CGS3) spectrum which goes out to $23.5 \mu \mathrm{m}$. These authors speculate that the high IRAS $25 \mu \mathrm{m}$ flux level might be due to a strong emission feature, at wavelengths longer than $\simeq 24 \mu \mathrm{m}$, i.e. the " 30 " $\mu \mathrm{m}$ feature. When we convolve the synthetic SED with the IRAS filter profile, the IRAS $25 \mu \mathrm{m}$ flux is indeed nicely reproduced by our model.

The excellent agreement, between the simple detachedshell model and the ISO and IRAS data, demonstrates that the "30" $\mu \mathrm{m}$ feature arises from the location of the detached shell. We conclude, that $\mathrm{MgS}$ grains are present in the detached shell of R Scl. We would like to emphasise that we do not attempted to further optimise the obtained model by varying the inner or outer edge of the dust shell. We have to make a rather crude assumption for the absorption level of the MgS grains below $1 \mu \mathrm{m}$ (Sect. 2.2). Therefore, this simple model does not warrant such a detailed fitting procedure. However, the correspondence between the model and observations, especially those probing different spatial scales, demonstrates that the $\mathrm{MgS}$ emission arises from roughly the same location as the molecular gas emission.

\subsection{Application to U Camelopardalis}

We also apply the model to U Cam. Compared to R Scl, this object has a much thinner CO shell (Table 1). Lindqvist et al. (1999) measured an inner radius of $5.5 \times 10^{16} \mathrm{~cm}$ with a 


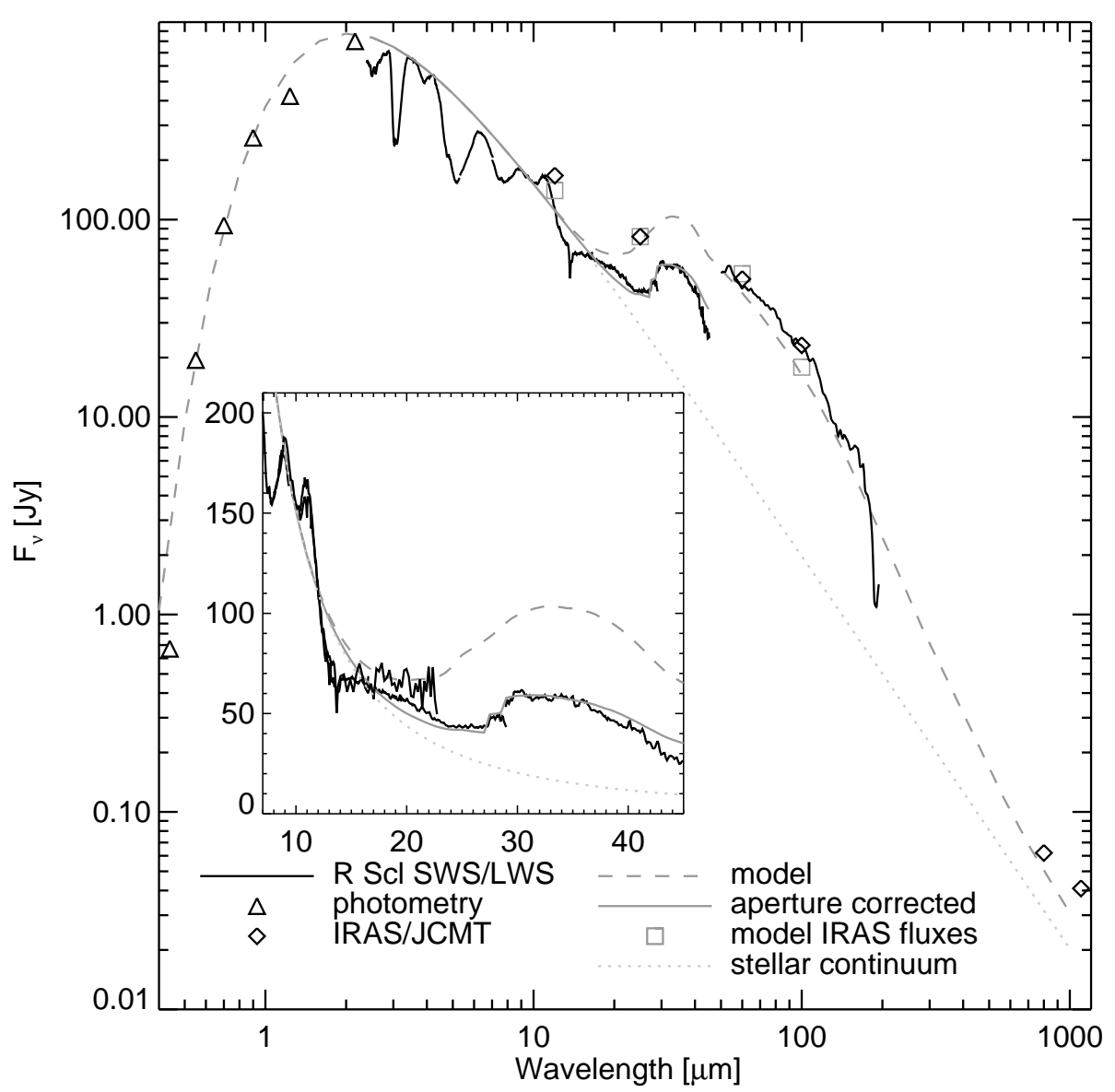

Fig. 7. The detached-shell model compared to the observations of $\mathrm{R} \mathrm{Scl}$ in black. The optical photometry is shown in triangles (Johnson et al. 1966). Between 2 and $45 \mu \mathrm{m}$ we show the SWS spectrum (Hony et al. 2002); between 45 and $200 \mu \mathrm{m}$ we show the LWS spectrum. The diamonds represent the IRAS measurements and the submillimetre points from Bagnulo et al. (1998). The dashed gray line is the model SED, taking identical parameters for the dust shell as derived for the $\mathrm{CO}$ shell by Schöier \& Olofsson (2001). The full gray line shows the model spectrum after correction for the limited size of the apertures of the SWS spectrograph. The dotted line represents the photospheric continuum. We also show the synthetic IRAS fluxes as derived from the model SED. The 25 and $60 \mu \mathrm{m}$ IRAS fluxes are in excellent agreement. The predicted flux level at longer wavelengths is slightly too low. The model explains the observations very well. In the inset, we show the fit to the " 30 " $\mu \mathrm{m}$ feature on a linear scale. We also show the IRAS/LRS spectrum, which starts to deviate from the SWS spectrum at the wavelength, where the model predicts a difference, due to the different beam-size of IRAS and SWS.
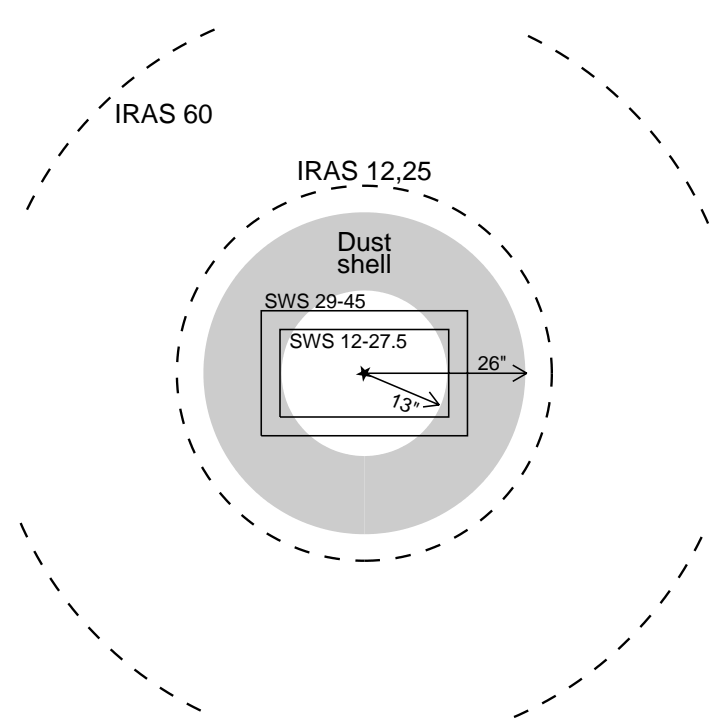

Fig. 8. The angular dimensions of the circumstellar shell of $\mathrm{R} \mathrm{Scl}$ compared with the regions probed by ISO/SWS and IRAS. The black rectangles indicate the dimensions of the two most relevant SWS apertures. The dashed circles give an indication of the regions corresponding to IRAS 12,25 and $60 \mu \mathrm{m}$ point source measurements. The circle for the IRAS $100 \mu \mathrm{m}$ filter lies outside the figure. The shell extends from $13^{\prime \prime}$ to $26^{\prime \prime}$, which corresponds to $7 \times 10^{16}-14 \times 10^{16} \mathrm{~cm}$ at a distance of $360 \mathrm{pc}$. The complete shell is probed by the IRAS measurements. The SWS instrument detects only a part of the shell radiation. Of course, the back and front side of the spherical shell do contribute to the SWS flux. thickness of only $1.1 \times 10^{16} \mathrm{~cm}$ at a distance of $500 \mathrm{pc}$. This corresponds to an outer radius of $9^{\prime \prime}$ and the shell fits entirely within the largest SWS aperture. With those values for the inner and outer radius of the shell the model cannot explain the IRAS and the ISO measurements simultaneously (Fig. 9). We find that the low IR flux levels, as measured by ISO/SWS, place a stringent constraint on the amount of dust located at the position of the $\mathrm{CO}$ shell. However, the IRAS fluxes clearly indicate that there is more dust present in the system. This dust is probably located even farther away from the star. As can be seen in Fig. 9, all the IRAS fluxes as well as the $800 \mu \mathrm{m}$ flux are under-predicted by the shell model. When we increase the shell dust mass, in order to agree with the IRAS 25 and $60 \mu \mathrm{m}$ flux levels, the longer wavelength fluxes are still very much under predicted by the relatively warm dust in the model. This indicates that the system contains cooler dust, which is probably located farther away. The latter conclusion is in qualitative agreement with the fact that the interferometric measurements of Lindqvist et al. (1999) retrieved about half of the total CO emission, measured with single dish observations (Neri et al. 1998). This is due to a more extended component of $\mathrm{CO}$ emission.

The situation in the case of U Cam is clearly more complex than for R Scl. The observations can not be explained by a single dust shell. The far-IR fluxes levels are dominated by dust located outside the detected CO shell. However, we stress that there is $\mathrm{MgS}$ present in the CO shell. The ISO/SWS instrument, which only probes the inner regions, detects a " 30 " $\mu \mathrm{m}$ feature. The temperature of the $\mathrm{MgS}$, as derived from the 


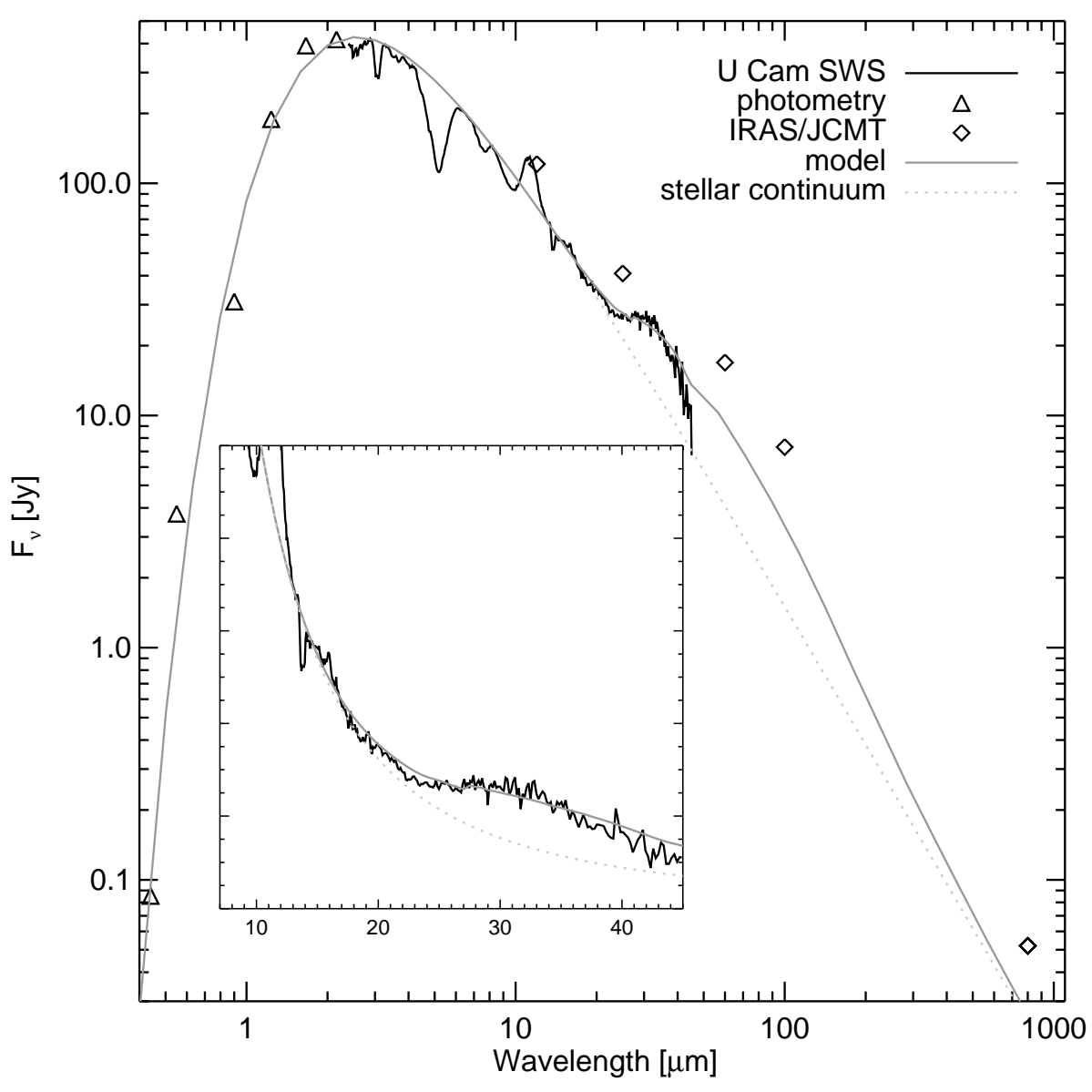

Fig. 9. The single detached-shell model applied to U Cam. Symbols are the same as in Fig. 7. We use the inner and outer radius and the gas mass of the $\mathrm{CO}$ shell and a $4 \% \mathrm{MgS}$ fraction. The model that fits the SWS observations best does not reproduce the IRAS flux levels. Clearly, the single dust shell model is not applicable and the IRAS excess is predominantly due to more extended emission than the CO shell. centroid position of the " 30 " $\mu \mathrm{m}$ feature in the ISO observation, yields a distance for the dust shell of $5.5 \times 10^{16} \mathrm{~cm}$ $\left(7.3^{\prime \prime}\right.$ at a distance of $500 \mathrm{pc}$ ). This distance is identical to the size of the detached CO shell. Using the gas mass of the shell of $1.4 \times 10^{-3} M_{\odot}$ as given by Schöier \& Olofsson (2001) and a dust mass of $2.4 \times 10^{-6} M_{\odot}$ from the best fitting model for the ISO/SWS data, we find a gas-to-dust mass ratio of $\Psi=580$.

We would like to point out that, in the two cases that we study here, the derived gas-to-dust mass ratio in the shell is relatively high (450 for $\mathrm{R} \mathrm{Scl}$ and 580 for $\mathrm{U} \mathrm{Cam}$ ). These numbers are much higher than the values between 200 and 250, which are commonly used for modelling carbon-rich AGB and post-AGB stars (e.g. Jura 1986; Meixner et al. 1997). However, the numbers we derive are in relatively good agreement with the high values of Schöier \& Olofsson (2001). One should clearly be cautious in applying a "standard" value, when determining the total shell masses for such systems based on the IR excess alone.

\subsection{Other warm carbon stars with the " 30 " $\mu \mathrm{m}$ feature}

There are a few more warm and optically bright carbon stars that exhibit a cool MgS feature in their SWS spectra. We do not model these stars here, mostly because of the lack of suitable molecular data to compare with. However, we do give a crude estimate of their shell radii. We calculate the angular radius of the star times the effective temperature squared from the bolometric magnitude given by Bergeat et al. (2002), using:

$$
\begin{aligned}
m_{\mathrm{bol}, \star} & =M_{\mathrm{bol}, \star}-2.5 \log _{10}\left(\left(\frac{10}{d}\right)^{2}\right)+M_{\mathrm{bol}, \odot}-M_{\mathrm{bol}, \odot} \\
& =M_{\mathrm{bol}, \odot}-5 \log _{10}\left(\left(\frac{R_{\star}}{R_{\odot}}\right)\left(\frac{T_{\star}}{T_{\odot}}\right)^{2}\left(\frac{10}{d}\right)\right) \Rightarrow \\
R_{\star, \operatorname{arcsec}} T_{\star}^{2} & =\left(\frac{\left(M_{\mathrm{bol}, \odot}-m_{\mathrm{bol}, \star}\right)}{5}\right)^{10} \frac{R_{\odot}}{10 \mathrm{AU}} T_{\odot}^{2}
\end{aligned}
$$

where $m_{\mathrm{bol}}$ and $M_{\mathrm{bol}}$ are the apparent and absolute bolometric magnitude and $R_{\star, \text { arcsec }}$ is the angular radius of the star. The measured values of the apparent bolometric magnitude and the centroid position of the MgS feature together with Eq. (2) and (3) yields the angular radius of the shell. The sources and the estimated radii are listed in Table 2. These sources all have much weaker " 30 " $\mu \mathrm{m}$ features than the feature observed in the SWS spectrum of R Scl, with a correspondingly lower signalto-noise ratio. At the same time, the estimated stellar continuum underlying the " 30 " $\mu \mathrm{m}$ feature becomes more influential on the derived feature profile. Based on this, we estimate an uncertainty in $\lambda_{\mathrm{C}, 30}$ of $\gtrsim 1 \mu \mathrm{m}$ and in the derived shell radii of at least a factor 2 .

\section{Conclusions and future prospects}

With the models that we have presented, we now have a tool to systematically investigate the influence of $\mathrm{MgS}$ on the 
Table 2. Estimated detached-shell radii $\left(R_{\text {shell }}\right)$ of warm carbon stars exhibiting a cool MgS feature in their SWS spectra, based on the relation in Eq. (2) between the "30" $\mu \mathrm{m}$ feature centroid position and the size of the shell.

\begin{tabular}{llcc}
\hline \hline Source & $m_{\text {bol }}{ }^{1}$ & $\lambda_{\mathrm{C}, 30}{ }^{2}[\mu \mathrm{m}]$ & $R_{\text {shell }}\left[{ }^{\prime \prime}\right]$ \\
\hline W Ori & 2.87 & 31.3 & 8 \\
S Cep & $3.15^{\dagger}$ & 31.2 & 6 \\
T Dra & 4.94 & 30.2 & 1.6 \\
V Cyg & $3.24^{\dagger}$ & 30.5 & 4 \\
RU Vir & $4.24^{\dagger}$ & 30.4 & 2 \\
\hline
\end{tabular}

${ }^{1}$ Bolometric magnitude from Bergeat et al. (2002). ${ }^{2}$ Centroid positions taken from Hony et al. (2002). ${ }^{\dagger}$ Average of the listed values.

IR spectra of carbon stars with detached shells. We find that, in the case of $\mathrm{R} \mathrm{Scl}$, the dust (containing $\mathrm{MgS}$ ) is co-spatial with the molecular gas. This shows that the dust present in the shell is indicative of C-rich chemistry and therefore the photosphere was $C$-rich at the time the shell was ejected. This rules against a scenario in which the shell is produced due to the transition from O-rich to $\mathrm{C}$-rich. We find that the shell around U Cam contains $\mathrm{MgS}$ as well, again demonstrating the C-based chemistry of the shell.

The prominence of the " 30 " $\mu \mathrm{m}$ feature and the broad wavelength range over which the $\mathrm{MgS}$ grains emit make this material an excellent tool for future studies of the circumstellar shells of carbon-rich evolved stars. In particular, we point out that, because of the temperature dependence of the $\mathrm{MgS}$ emission profile, some of the usual ambiguity in interpreting SEDs can be lifted, as we can constrain the location of the shell by means of the " 30 " $\mu \mathrm{m}$ feature. The low-resolution longwavelength mode of the IRS instrument (Roellig et al. 1998) on board SIRTF (Gallagher et al. 2003) will be sensitive enough to easily detect the $\mathrm{MgS}$ feature in carbon stars located in the SMC or LMC. This will eliminate the distance uncertainty, which usually troubles the derivation of the shell parameters of galactic carbon stars and therefore allows a more statistical approach for studying the occurrence rate and shell masses of such detached shells.

Another tantalising prospective is offered by the FORECAST instrument (Keller et al. 2000) for the SOFIA observatory (Becklin 1997). With this camera it will be feasible to directly image the shells at the wavelengths where the $\mathrm{MgS}$ is emitting. The shells discussed here are all easily resolved with the good angular resolution $\left(<5^{\prime \prime}\right)$ of the large SOFIA mirror. The several bandpasses in the " 30 " $\mu \mathrm{m}$ feature will allow us to study the $\mathrm{MgS}$ abundance and temperature as a function of distance to the carbon star to an unprecedented level of detail.

Acknowledgements. The authors thank Frank Molster, Jacco van Loon and Rens Waters for interesting and very constructive discussions. We thank Coralie Neiner for careful reading of the manuscript. JB acknowledges financial support from the EC-RTN on "The Formation and Evolution of Young Stellar Clusters" (RTN1999-00436, HPRN-CT-2000-00155). This research has made use of the SIMBAD database, operated at CDS, Strasbourg, France.
This research has made use of NASA's Astrophysics Data System Bibliographic Services. This research has made use of the VizieR catalogue access tool, CDS, Strasbourg, France.

\section{References}

Alksnis, A., Balklavs, A., Dzervitis, U., et al. 2001, Baltic Astron., 10, 1

Bagnulo, S., Doyle, J. G., \& Andretta, V. 1998, MNRAS, 296, 545

Bagnulo, S., Skinner, C. J., Doyle, J. G., \& Camphens, M. 1997, A\&A, 321, 605

Becklin, E. E. 1997, in The Far Infrared and Submillimetre Universe, 201

Begemann, B., Dorschner, J., Henning, T., Mutschke, H., \& Thamm, E. 1994, ApJ, 423, L71

Beichman, C. A., Neugebauer, G., Habing, H. J., Clegg, P. E., \& Chester, T. J. 1988, in NASA RP-1190, 1, 0

Bergeat, J., Knapik, A., \& Rutily, B. 2001, A\&A, 369, 178

Bergeat, J., Knapik, A., \& Rutily, B. 2002, A\&A, 390, 967

Bouwman, J. 2001, Ph.D. Thesis, University of Amsterdam

Bouwman, J., de Koter, A., van den Ancker, M. E., \& Waters, L. B. F. M. 2000, A\&A, 360, 213

Bujarrabal, V., \& Cernicharo, J. 1994, A\&A, 288, 551

Chan, S. J., \& Kwok, S. 1988, ApJ, 334, 362

Dayal, A., Hoffmann, W. F., Bieging, J. H., et al. 1998, ApJ, 492, 603

de Graauw, T., Haser, L. N., Beintema, D. A., et al. 1996, A\&A, 315 , L49

Egan, M. P., Kwok, S., Leung, C. M., \& Price, S. D. 1996, A\&A, 308, 738

Feautrier, P. 1964, C. R. Acad. Sci. Paris, 258, 3189

Forrest, W. J., Houck, J. R., \& McCarthy, J. F. 1981, ApJ, 248, 195

Gallagher, D. B., Irace, W. R., \& Werner, M. W. 2003, in IR Space Telescopes and Instruments, ed. J. C. Mather, Proc. SPIE, 4850, 17 Goebel, J. H., \& Moseley, S. H. 1985, ApJ, 290, L35

González Delgado, D., Olofsson, H., Schwarz, H. E., Eriksson, K., \& Gustafsson, B. 2001, A\&A, 372, 885

Hony, S., Tielens, A. G. G. M., Waters, L. B. F. M., \& de Koter, A. 2003, A\&A, 402, 211

Hony, S., Waters, L. B. F. M., \& Tielens, A. G. G. M. 2002, A\&A, 390,533

Hrivnak, B. J., Volk, K., \& Kwok, S. 2000, ApJ, 535, 275

Ivezic, Z., \& Elitzur, M. 1995, ApJ, 445, 415

Izumiura, H., \& Hashimoto, O. 2000, Advances in Space Research, 25,2205

Izumiura, H., Hashimoto, O., Kawara, K., Yamamura, I., \& Waters, L. B. F. M. 1996, A\&A, 315, L221

Izumiura, H., Kester, D. J. M., De Jong, T., et al. 1995, Ap\&SS, 224, 495

Izumiura, H., Waters, L. B. F. M., De Jong, T., et al. 1997, A\&A, 323, 449

Izumiura, H., Waters, L. B. M. F., de Jong, T., Loup, C., \& Hashimoto, O. 2000, in The Carbon Star Phenomenon, ed. R. F. Wing, IAU Symp., 177, 415

Johnson, H. L., Iriarte, B., Mitchell, R. I., \& Wisniewskj, W. Z. 1966, Communications of the Lunar and Planetary Laboratory, 4, 99

Joint IRAS Science Working Group. 1988, in IRAS Point Source Catalog (1988), 0

Jura, M. 1986, ApJ, 303, 327

Jura, M., Chen, C., \& Werner, M. W. 2000, ApJ, 544, L141

Keller, L. D., Herter, T. L., Stacey, G. J., et al. 2000, in Airborne Telescope Systems, ed. R. K. Melugin, \& H.-P. Roeser, Proc. SPIE, 4014, 86

Kessler, M. F., Steinz, J. A., Anderegg, M. E., et al. 1996, A\&A, 315, L27 
Kwok, S., Volk, K., \& Hrivnak, B. J. 2002, ApJ, 573, 720

Lattimer, J. M., Schramm, D. N., \& Grossman, L. 1978, ApJ, 219, 230 Lindqvist, M., Olofsson, H., Lucas, R., et al. 1999, A\&A, 351, L1

Lodders, K., \& Fegley, B. 1999, in Asymptotic Giant Branch Stars, IAU Symp., 191, 279

Meixner, M., Skinner, C. J., Graham, J. R., et al. 1997, ApJ, 482, 897 Mihalas, D. 1978, Stellar atmospheres /2nd edition/ (San Francisco, W. H. Freeman and Co., 1978. 650 p.)

Neri, R., Kahane, C., Lucas, R., Bujarrabal, V., \& Loup, C. 1998, A\&AS, 130, 1

Nuth, J. A., Moseley, S. H., Silverberg, R. F., Goebel, J. H., \& Moore, W. J. 1985, ApJ, 290, L41

Olofsson, H., Bergman, P., Eriksson, K., \& Gustafsson, B. 1996, A\&A, 311, 587

Omont, A. 1993, in Astronomical Infrared Spectroscopy: Future Observational Directions, ASP Conf. Ser., 41, 87

Preibisch, T., Ossenkopf, V., Yorke, H. W., \& Henning, T. 1993, A\&A, 279, 577

Roellig, T. L., Houck, J. R., Van Cleve, J. E., et al. 1998, in Infrared Astronomical Instrumentation, ed. A. M. Fowler, Proc. SPIE, 3354, 1192
Schöier, F. L., \& Olofsson, H. 2001, A\&A, 368, 969

Schröder, K.-P., Winters, J. M., Arndt, T. U., \& Sedlmayr, E. 1998, A\&A, 335, L9

Simis, Y. J. W., Icke, V., \& Dominik, C. 2001, A\&A, 371, 205

Steffen, M., \& Schönberner, D. 2000, A\&A, 357, 180

Szczerba, R., Henning, T., Volk, K., Kwok, S., \& Cox, P. 1999, A\&A, 345, L39

van der Veen, W. E. C. J., \& Habing, H. J. 1988, A\&A, 194, 125

Waters, L. B. F. M., Loup, C., Kester, D. J. M., Bontekoe, T. R., \& De Jong, T. 1994, A\&A, 281, L1

Waters, L. B. F. M., Molster, F. J., Hony, S., et al. 2000, Thermal Emission Spectroscopy and Analysis of Dust, Disks, and Regoliths, ASP Conf. Ser., 196, 3

Willems, F. J., \& de Jong, T. 1988, A\&A, 196, 173

Yamamura, I., De Jong, T., Justtanont, K., Cami, J., \& Waters, L. B. F. M. 1998, Ap\&SS, 255, 351

Yamamura, I., Onaka, T., Kamijo, F., Izumiura, H., \& Deguchi, S. 1993, PASJ, 45, 573

Young, K., Phillips, T. G., \& Knapp, G. R. 1993, ApJS, 86, 517

Zuckerman, B. 1993, A\&A, 276, 367 